\title{
Multiple regenerative effects in cutting process and nonlinear oscillations
}

\author{
Xianbo Liu • Nicholas Vlajic • Xinhua Long • \\ Guang Meng • Balakumar Balachandran
}

Received: 4 September 2013 / Revised: 15 February 2014 / Accepted: 27 February 2014 / Published online: 18 March 2014

(C) Springer-Verlag Berlin Heidelberg 2014

\begin{abstract}
A turning process paradigm is considered to study multiple regenerative effects in cutting operations. The workpiece is considered to be a spatially continuous element, while the cutter is modeled as a discrete-parameter element. The resulting system is described by a combined partial differential equation-ordinary differential equation (ODE) model with a surface function that is used for updating the workpiece. The time delay in this model is allowed to be any integer multiple of the tooth-pass period. Analysis of this system reveals that the loss of contact between the workpiece and the cutter results in two principal features, namely, a non-smooth cutting force and multiple regenerative effects. The model of the spatially continuous work piece is cast into a system of ODEs through the semi-discretization method. Subsequent analysis results in a high-dimensional, non-smooth discrete-time map. Iterations of this mapping show that the time delay can vary in a wide range, and due to the multiple regenerative effect, this delay can be as high as ten times the constant delay value. Through parametric studies, it is learned that the system can exhibit stable cutting behavior, as well as periodic, quasi-periodic, chaotic and hyperchaotic behavior. With the choice of the non-dimensional cutting coefficient as a control parameter, bifurcations of the system responses are examined. The system is observed to possess rich dynamics, including multiple solutions. Supported by computations of correlation
\end{abstract}

\footnotetext{
X. Liu $\cdot$ X. Long $\cdot$ G. Meng

State Key Laboratory of Mechanical System and Vibration, School of Mechanical Engineering, Shanghai Jiao Tong University, Shanghai 200240, China

N. Vlajic $\cdot$ B. Balachandran $(\bowtie)$

Department of Mechanical Engineering, University of Maryland, College Park, MD 20742, USA

e-mail: balab@umd.edu
}

dimension, Kaplan-Yorke dimension, and Lyapunov spectrum, limit cycle, torus, chaotic, and hyperchaotic attractors are observed to be present in the considered parameter range. The findings can help further our understanding of multiple regenerative effects in cutting and drilling operations.

Keywords Chaos and hyperchaos - Cutting process . Non-smooth system $\cdot$ Regenerative effects .

Semi-discretization - Time delay

\section{Introduction}

In material removal operations, such as boring, turning, milling, and oil and gas drilling operations, time-delay effects due to regenerative cutting have been extensively studied. As a result of the different studies, it is known that a primary cause of the instability in these systems is tied to timedelay effects (e.g., [1-19]). Two typical cutting operations, namely, a turning operation for metal cutting and a drilling operation used for the retrieval of petrochemicals, are illustrated in Figs. 1a, b, respectively. In both of these operations, the instantaneous chip thickness $\delta$ is determined by both the current and previous position of the cutter or drill bit. In cutting operations, this dependence on the current and previous states is referred to as the regenerative cutting effect, and this effect is captured by introducing a time delay into the corresponding models. The representative cutting system, which is governed by delay differential equations (DDEs), is prone to self-excited vibrations. In metal cutting systems, chatter vibrations may occur due to regenerative cutting and lead to a wavy surface finish on the workpiece (e.g., [20]) or even poor machining precision. In oil and gas drilling operations, instabilities originating from the regenerative effect may lead to undesirable motions such as stick-slip vibrations and bit 
(a)

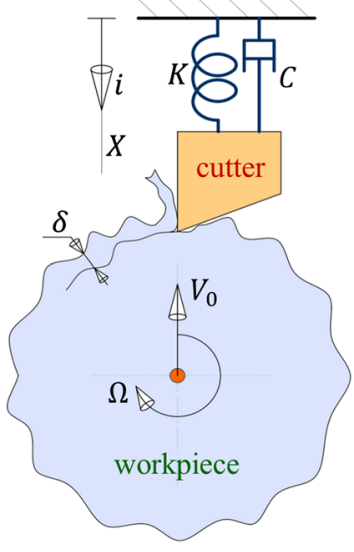

(b)

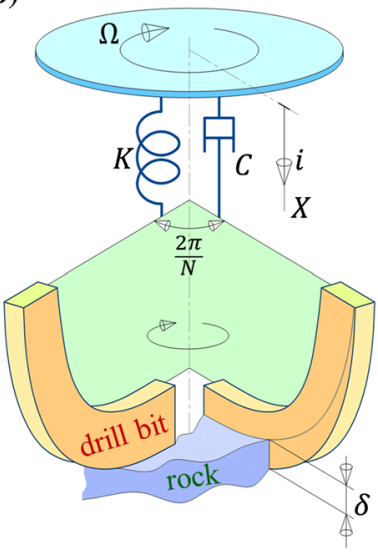

Fig. 1 Systems with regenerative effects: a turning process in metal cutting and $\mathbf{b}$ drilling process in oil and gas exploration

bounce which can both cause excessive tool wear and/or even expensive drill-bit failures [11,19].

Furthermore, a regenerative cutting process may be subject to nonlinear effects such as thickness-dependent cutting forces $[6,7,12,13,15]$, dry friction $[11,17,18,21]$, loss of contact [6-8, 11,12,15,17-19], nonlinear structural effects [9], and varying time-delay effects $[6,11,14,19]$. Due to the nonlinearities, bifurcations and chaotic motions can occur and the system dynamics can become complex. To understand this dynamics, Grabec [20] presented a model with nonlinear dependence of the cutting force on both velocity and chip thickness. Loss of contact is also taken into consideration and the results show that the system exhibits chaotic motions. Stépán [7] presented a single degree-offreedom (DOF) model with a nonlinear cutting force based on the so-called three-quarter rule. In this model, both the long and short regenerative effects, which introduce distributed time delays into the system, are taken into consideration. Tlusty and Ismail [3] introduced a nonlinearity due to the loss of contact between the cutter and workpiece. With this effect, the instantaneous chip thickness is determined by the current and three preceding positions of the cutter. This is the underlying concept of the so called multiple regenerative cutting which may introduce an integer multiple of the time delay $\left(\tau_{0}, 2 \tau_{0}, 3 \tau_{0}, \ldots\right)$ into the system. Balachandran and Zhao $[6,10]$ developed a unified nonlinear model to study the dynamics of a typical milling process. In this model, feed rate effects and multiple regenerative effects are taken into consideration. Based on this model, the stability boundary is determined by using time domain simulations. Recently, Litak et al. [15,16] use a statistical 0-1 test to identify chaotic motions in a nonlinear turning process based on the model from a previous study [7]. Banihasan and Bakhtiari-Nejad [22] investigated chaotic motions in high-speed milling based on a model presented in earlier studies $[6,10]$. They considered segmental multiple regener-

ative effects and used Lyapunov exponents to identify chaotic behavior. Considering dynamics of turning process, Wahi and Chatterjee [23] developed an ODE-PDE model, in which both the multiple-regenerative effects and the nonlinear cutting force model based on three-quarter power law are taking into consideration. Dombovari et al. [24] approximated the non-smooth delay differential algebraic equation with a smooth function. The standard numerical continuation package DDE-BITTOOL is used to analyze bifurcations of the system responses. It is worth noting that, in all of the studies cited above, the multiple regenerative effect is not the sole focus of the work. Hence, here, a study focusing on multiple regenerative effects is presented and the corresponding nonlinear motions are examined.

This paper is organized as follows. In Sect. 2, an original model made up of one PDE and one ODE is constructed to investigate the multiple regenerative effect. Following that, an algorithm to study this PDE-ODE system is developed. A discrete-time map is obtained based on the semi-discretization method. In Sect. 3, numerical studies are conducted to illustrate the multiple regenerative effect and the resulting nonlinear oscillations. The observed highdimensional chaotic behavior is analyzed by using Poincaré sections, Lyapunov spectrum, and dimension calculations. Finally, based on the findings, conclusions are drawn and presented in Sect. 4.

\section{Modeling and system reduction}

\subsection{PDE-ODE model for multiple regenerative cutting system}

In order to focus on only the nonlinear dynamics resulting from the multiple regenerative effect, a simplified model with one degree-of-freedom is considered for a cutting system. As shown in Fig. 1, there are many similarities between turning and drilling operations. Neglecting the state-dependent delay due to the torsional oscillations $[11,18]$, the equations of motion of these two systems in the $i$ direction will have the same form but with different coefficients. In this article, a turning system is used as an illustrative example and the parameters are chosen to be compatible with drilling operations. Here, the workpiece is assumed to be driven at a constant angular speed $\Omega$ and a constant feed speed $V_{0}$. By using the equilibrium position of the mass-spring-damper system as the zero position of $X$, the equation of motion of the cutter or drill bit is an ODE of the form

$M \ddot{X}+C \dot{X}+K X=F(X, U, t)$

where, $M, C$, and $K$ are the equivalent mass, damping and stiffness parameters of the cutting system, respectively. The 

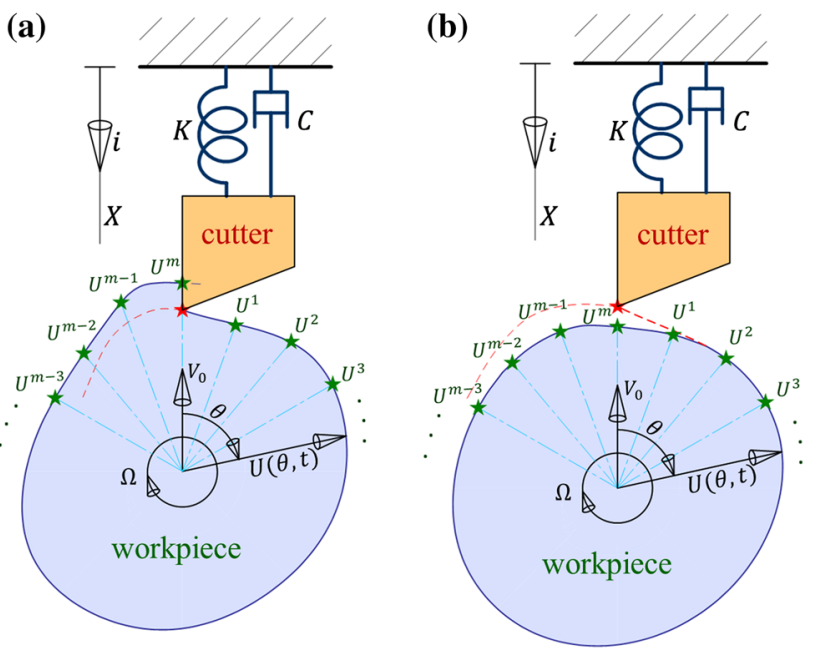

Fig. 2 Multiple regenerative model and the discretization of workpiece: a cutting during contact and $\mathbf{b}$ cutting does not occur during loss of contact

quantity $F(X, U, t)$ is the cutting force, which is a function of $X, U$, and time $t$. As depicted in Fig. 2, $X(t)$ is the current position of the cutter measured along the $i$ direction . The quantity $U(\theta, t)$, which is the so called surface height function [24], is the instantaneous radius of the workpiece at angular position $\theta \in[0,2 \pi]$ and at time $t$. Physically, $U$ describes the surface of workpiece. As the workpiece moves at a constant feed rate $V_{0}$, the cutting depth, which is a function of $X$ and $U$, can be written as

$\delta(X, U, t)=X(t)-\left[R_{0}-V_{0} t-U(2 \pi, t)\right]$

The quantity $R_{0}$ is the position of the rotating center of the workpiece at time $t=0$ along the $i$ direction. Since the cutter can loose contact with the surface, material is removed from the workpiece only if $\delta>0$. Consequently, the cutting force is a non-smooth function with respect to $\delta$ and can be written as

$F(X, U, t)=\bar{F}(\delta)= \begin{cases}-K_{c} d_{a} \delta, & \delta>0 \\ 0, & \delta \leq 0\end{cases}$

The quantity $d_{a}$ is the axial depth of cut and $K_{c}$ is the cutting coefficient. Here, the cutting force is modeled as a linear function of the instantaneous depth of cut.

Considering an arbitrary fixed point $\left(\theta=\theta_{0}+\Omega t\right)$ on the workpiece surface that is being rotated at a constant angular speed $\Omega$, the radius of this point $U(\theta(t), t)$ should be a constant when $\theta(t)=\theta_{0}+\Omega t \in[0,2 \pi]$. Thus, it follows that

$\frac{d U(\theta(t), t)}{d t}=\frac{\partial U}{\partial t}+\Omega \frac{\partial U}{\partial \theta}=0, \quad \theta \in[0,2 \pi]$

Equation (4) is the updating rule for the workpiece surface. As shown in Fig. 2a, when the cutter is removing material, the radius at the contact point $U(0, t)$ should be relative to the position of the cutter $X(t)$. When the cutter looses contact with the workpiece, the surface of the workpiece should be continuous and independent of $X(t)$. Thus, the boundary condition for the PDE (4) is written as

$U(0, t)= \begin{cases}R_{0}-V_{0} t-X(t), & \delta(t)>0 \\ U(2 \pi, t), & \delta(t) \leq 0\end{cases}$

From Eq. (5), it is clear that the boundary condition for the PDE switches depending on the contact between the cutter and workpiece. Instead of using a minimum function as in reference [23], here, a switching boundary condition is used to introduce the multiple regenerative effects.

The mixed PDE-ODE system given by Eqs. (1)-(5) has a steady-state response (trivial solution) during which cutting occurs without the cutter vibrations. This steady-state solution can be written as

$$
\left\{\begin{array}{l}
X_{0}(t)=-\frac{2 \pi K_{c} d_{a} V_{0}}{K \Omega} \\
\delta_{0}(t)=\frac{2 \pi V_{0}}{\Omega} \\
U_{0}(\theta, t)=R_{0}+\frac{V_{0}}{\Omega} \theta-V_{0} t+\frac{2 \pi K_{c} d_{a} V_{0}}{K \Omega}
\end{array}\right.
$$

To simplify the equations and reduce the number of parameters, the equations are cast in non-dimensional form by using following dimensionless variables:

$\left\{\begin{array}{l}\tau=\omega_{0} t \\ x=\frac{X-X_{0}}{\delta_{0}} \\ u=\frac{U_{0}-U}{\delta_{0}}\end{array}\right.$

The term $\omega_{0}=\sqrt{\frac{K}{M}}$ is the radian natural frequency of the cutting system. On substituting Eq. (7) into Eqs. (1)-(5), the dimensionless equations of motion are obtained as

$$
\left\{\begin{array}{l}
\text { if } u(2 \pi, t)-x<1 \quad \cdots \cdots \text { contact case } \\
\quad \ddot{x}+2 \zeta \dot{x}+x=\kappa[u(2 \pi, \tau)-x] \\
\quad \dot{u}+\omega u^{\prime}=0 \\
\quad \text { boundary condition }: u(0, \tau)=x \\
\text { else } \quad \cdots \cdots \text { loss of contact case } \\
\quad \ddot{x}+2 \zeta \dot{x}+x=\kappa \\
\quad \dot{u}+\omega u^{\prime}=0 \\
\text { boundary condition }: u(0, \tau)=u(2 \pi, \tau)-1
\end{array}\right.
$$

The operation $(\cdot)$ denotes the derivative or partial derivative with respect to the dimensionless time $\tau$, and $(\cdot)^{\prime}$ denotes the partial derivative with respect to the spatial parameter $\theta$. The frequency ratio of the drive speed $\Omega$ to the natural 
frequency $\omega_{0}$ is denoted by $\omega$. The quantity $\zeta$ is the damping ratio and $\kappa$ is the dimensionless parameter with respect to the cutting coefficient $K_{c}$ and axial depth of cut $d_{a}$. All of these non-dimensional parameters can be written as

$$
\left\{\begin{array}{l}
\omega=\frac{\Omega}{\omega_{0}} c \\
\zeta=\frac{{ }^{2} \sqrt{M K}}{K} \\
\kappa=\frac{K_{c} d_{a}}{K}
\end{array}\right.
$$

Equation (8) represents a coupled ordinary-partial differential system with switching boundary conditions. During the contact case, the ODE is coupled with the PDE through the cutting force term, while the PDE is coupled with the ODE through the boundary condition. In the loss of contact case, the ODE is a free oscillating system with damping, while the PDE is a simple shift (rotating) system. There is no coupling between the ODE and PDE. However, the boundary condition for the PDE is quite different from that in the contact case. In this boundary condition, the surface function $u(\theta, \tau)$ will keep on decreasing until $u(2 \pi, \tau)-x<1$; thus, the boundary condition can help break the status of loss of contact and the cutter can engage in cutting again. Time-delay effects are introduced into the cutting system by the PDE used for the updating of the workpiece surface. In addition, the switching boundary condition will introduce non-smooth cutting forces and result in multiple regenerative cutting. If the switching boundary condition and the loss of contact are not considered, then Eq. (8) degenerates to the cutting model without the multiple regenerative effect, which has been studied quite extensively in the literature (e.g., $[7,9,11-15,17,18]$ ).

\subsection{From PDE-ODE system to nonlinear map based on semi-discretization method}

In order to solve this ordinary-partial differential system numerically, the partial differential equation is discretized into a series of ordinary differential equations based on the semi-discretization method proposed by Insperger and Stépán $[25,26]$. The workpiece surface is segmented by first discretizing $\theta$ into $m$ spatial segments $\left(\theta^{1}, \theta^{2}, \cdots \theta^{m}\right)$, as shown in Fig. 2. The angle interval $\left[\theta^{i}, \theta^{i+1}\right]$ has a constant length $\Delta \theta$, which is given by

$\Delta \theta=\frac{2 \pi}{m}$

It is noted that $m+0.5$ is used instead of $m$ to improve the accuracy in earlier studies [25,26]; however, here, $m+0.5$ leads to poor accuracy in the numerical results because of the multiple regenerative effect (time-varying delay). In a similar manner to the $\theta$ discretization, the dimensionless time $\tau$ is discretized into $\tau_{1}, \tau_{2}, \tau_{3} \cdots$ and $\left[\tau_{i}, \tau_{i+1}\right]$ is a time interval of constant length $\Delta \tau$, which is given by

$\Delta \tau=\frac{\Delta^{\theta}}{\omega}=\frac{\tau_{0}}{m}=\frac{2 \pi}{\omega m}$

The quantity $\tau_{0}=\frac{2 \pi}{\omega}$ is the period of rotation of the workpiece. During a time step of $\Delta \tau$, the workpiece rotates by an angular displacement step of $\Delta \theta$. As a result of the discretization, $u(\theta, t)$ is constant in each angle interval $\left[\theta^{i}, \theta^{i+1}\right]$. Furthermore, $u_{j}^{i}$ is the resampling in space and time of $u(\theta, t)$, wherein, the indices $i$ and $j$ are the spatial index and temporal index, respectively. Then, the discretization of $u(\theta, t)$ takes the explicit form

$$
\begin{aligned}
u(\theta, \tau) & \approx u\left(\theta^{i}, \tau_{j}\right)=u_{j}^{i}, \\
& \text { for } \theta \in\left(\theta^{i-1}, \theta^{i}\right] \text { and } \tau \in\left(\tau_{i-1}, \tau_{i}\right]
\end{aligned}
$$

Equation (8) is linear in each time interval $\left[\tau_{i}, \tau_{i+1}\right]$. Hence, a piecewise linear mapping system corresponding to Eq. (8) can be obtained based on the zeroth-order semidiscretization method $[25,26]$ as follows:

$$
\left\{\begin{array}{c}
\text { if } u_{i}^{m}-x_{i}<1 \quad \cdots \cdots \text { contact case } \\
y_{i+1}=C_{1} y_{i} \\
\text { else } \quad \cdots \cdots \text { loss of contact case } \\
y_{i+1}=C_{2} y_{i}+D_{2}
\end{array}\right.
$$

The vector $y_{i}$ has a length $m+2$ and the components are given by

$y_{i}=\left(\begin{array}{llllll}x_{i} & \dot{x}_{i} & u_{i}^{1} & u_{i}^{2} & u_{i}^{3} \cdots u_{i}^{m-1} & u_{i}^{m}\end{array}\right)^{T}$

The superscript $(\cdot)^{T}$ denotes the transpose operation of the associated matrix or vector. The matrices $C_{1}$ and $C_{2}$ are square matrices with $m+2$ dimension, while $D_{2}$ is a vector of dimension $m+2$. Representations of these matrices and vectors take the following forms:

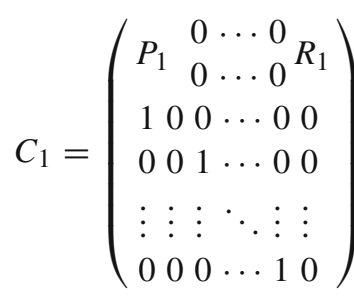

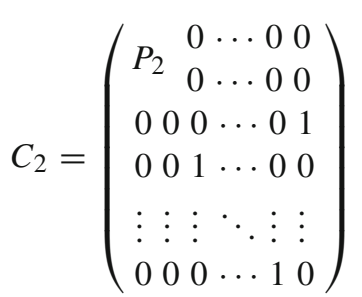




$$
D_{2}=\left(\begin{array}{lllllll}
R_{2}^{T} & -1 & 0 & 0 & \cdots & 0 & 0
\end{array}\right)^{T}
$$

The submatrices $P_{1}, P_{2}, R_{1}$, and $R_{2}$ can be written as

$P_{i}=e^{A_{i} \Delta \tau}, \quad R_{i}=\left(P_{i}-\mathbf{I}\right) A_{i}^{-1} B, \quad i=1$ or 2

where $e^{A_{i} \Delta \tau}$ is the matrix exponential of $A_{i} \Delta \tau$. The superscript $(\cdot)^{-1}$ denotes the inverse of matrix, and $\mathbf{I}$ is the identity matrix. Matrices $A_{i}$ and $B$ are the coefficient matrices that can be constructed from the matrix form of the ordinary differential equation in Eq. (8). They can be written as

$$
\begin{aligned}
A_{1} & =\left(\begin{array}{cc}
0 & 1 \\
-1-\kappa & -2 \zeta
\end{array}\right) \\
A_{2} & =\left(\begin{array}{cc}
0 & 1 \\
-1 & -2 \zeta
\end{array}\right) \\
B & =\left(\begin{array}{l}
0 \\
\kappa
\end{array}\right)
\end{aligned}
$$

For brevity, here, the authors have just provided the necessary results to make sure that a reader can reproduce the results of this paper. To help with the full derivation of Eqs.(13-21), one is referred to reference [26]. Equation (13) is a nonlinear mapping procedure for the multiple regenerative cutting system, and it can be conceptually be visualized as a higher-dimensional tent mapping (e.g., [27]). At the beginning of each iteration, the value of $u_{i}^{m}-x_{i}$ needs to be calculated to determine the initial point for the piecewise linear mapping. The nonlinear dynamics of this $m+2$ dimensional system is studied in the next section.

\section{Multiple regenerative cutting effect, nonlinear responses, and route to chaos}

\subsection{Numerical studies: tool responses, workpiece surface finish, and delay variation}

The nonlinear multiple regenerative cutting model given by Eq. (8) or (13) can be used to model both a metal cutting operation and an oil and gas drilling operation with appropriate choice of parameters. A linear model, which is a degenerate form of Eq. (8), can be obtained by neglecting both the nonsmooth cutting force and the multiple regenerative effect. This degenerate form can be written as

$\ddot{x}+2 \zeta \dot{x}+x=\kappa\left[x\left(\tau-\tau_{0}\right)-x\right]$

For both metal cutting operations and oil and gas drilling operations, the damping ratio $\zeta$ ranges from 0.02 to 0.1 $[7,16,18]$. Furthermore, in metal cutting operations, the frequency ratio $\omega$ is less than 3 , and the dimensionless cutting coefficient $\kappa$ is related to the cutter, workpiece material,

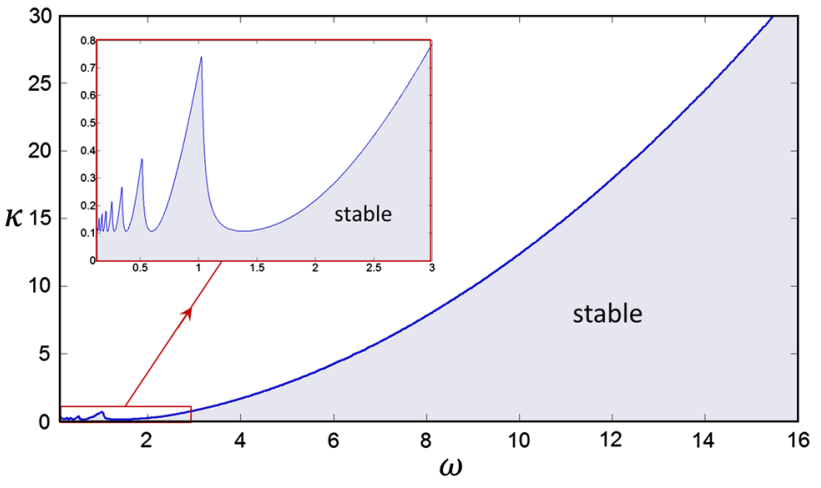

Fig. 3 Stability boundary in the parameter space of the dimensionless cutting coefficient and dimensionless drive speed for the linear cutting model (22). The stable cutting region is shaded

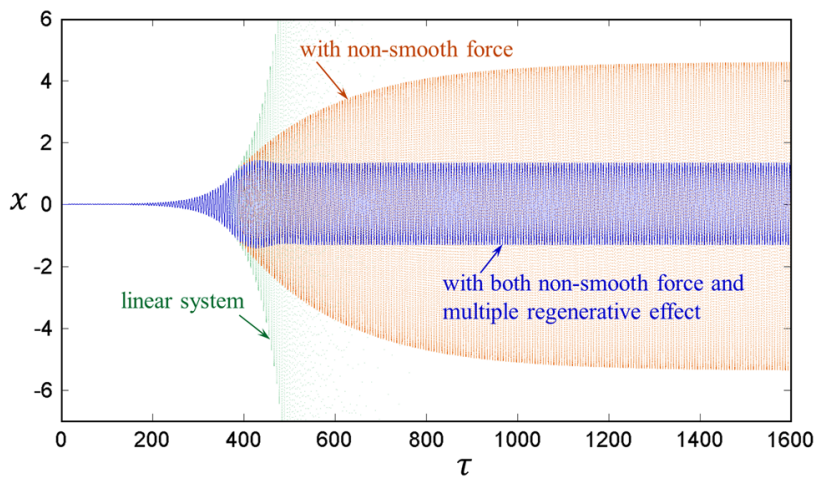

Fig. 4 Tool responses obtained from linear and nonlinear models for $\omega=0.4$ and $\kappa=0.3$

and axial depth of cut. In typical oil and gas drilling operations, the frequency ratio (tooth-pass frequency to natural frequency) ranges from zero to more than twenty due to the extremely low natural frequencies of the drill string system. The coefficient $\kappa$ which is related to the drill bit and rock stratum can range from tens to hundreds [28]. Here, as a representative case, the authors choose $\zeta=0.05$. Next, the stability analysis for the linear system is carried out and the stability lobe structure is shown in the expanded section of Fig. 3 for $\omega \in[0,3]$. For any parameter values inside the stable region, distrubances will decay and the system response will converge to zero and there will be stable cutting. On the other hand, for any parameters outside the stable region, the system response will diverge until the cutter looses contact with the workpiece and nonlinear oscillations follow. The focus of the rest of this section is on this unstable region and the associated nonlinear oscillations.

The effects of the non-smooth cutting force and multiple regenerative effect on the system response are illustrated in Fig. 4. For $\omega=0.4$ and $\kappa=0.3$, the response obtained from the linear model (22) is in the unstable region, and this response diverges in time. The response obtained from the nonlinear model, in which the non-smooth cutting 

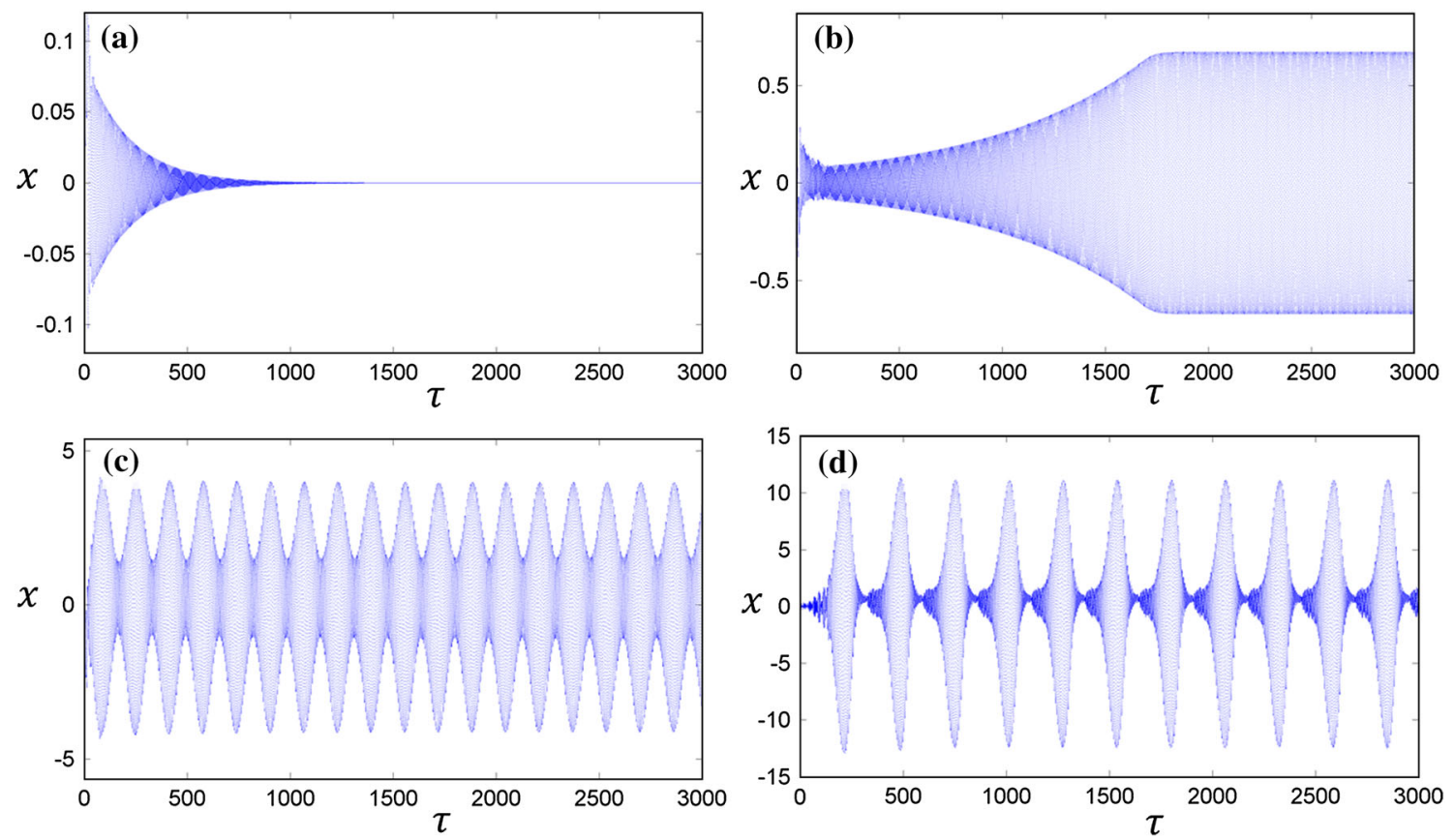

Fig. 5 Tool response in the presence of multiple regenerative cutting for $\omega=0.4$ : a $\kappa=0.1$, stable cutting; $\mathbf{b} \kappa=0.12$, periodic motion at the critical point of loss of contact; $\mathbf{c} \kappa=0.42$, quasi-periodic motion with loss of contact effects; and $\mathbf{d} \kappa=0.7$, chaotic motion
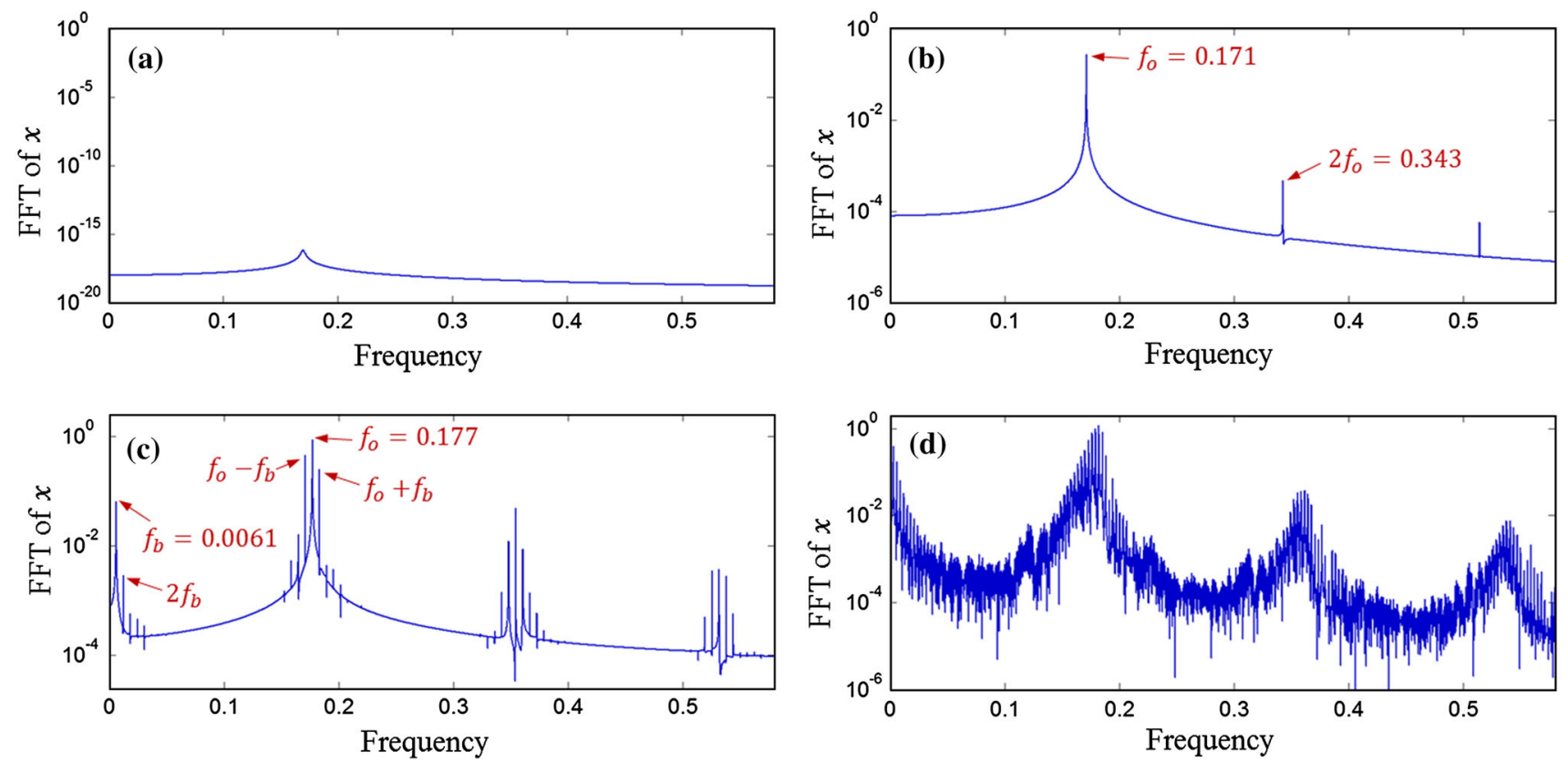

Fig. 6 Fourier transform of the signals in Fig. 5 for $\omega=0.4$ : $\mathbf{a} \kappa=0.1 ; \mathbf{b} \kappa=0.12 ; \mathbf{c} \kappa=0.42$; and $\mathbf{d} \kappa=0.7$

force is taken into consideration but the switching boundary condition is neglected, exhibits characteristics of limit-cycle motions. The combined model with both the non-smooth cutting force and multiple regenerative effect also leads to a limit cycle but with a smaller amplitude. Furthermore, simulations were carried out at a driving speed ratio $\omega=0.4$, which is a low driving speed for metal cutting systems. Noting that the mapping iteration Eq. (13) is numerically stable and accurate for $m>200$; here, the authors choose $m=600$ for the number of iterations for the remainder of this work.

Time histories of the tool response obtained for different values of the coefficient $\kappa$ are shown in Fig. 5, and the corresponding Fourier transforms of the steady-state signals from Fig. 5 are shown in Fig. 6. The corresponding updating of 
Fig. 7 Trajectory of cutting edge (dashed line) and the updating of workpiece surface (thick solid lines) at $\omega=0.4$ : a $\kappa=0.1, \mathbf{b} \kappa=0.12, \mathbf{c}$ $\kappa=0.42$, and $\mathbf{d} \kappa=0.7$. The dashed line denotes the loss of contact between the cutter and the workpiece. The starting points of the cutter on the workpiece are marked with triangles, while the ending points are marked with round dots
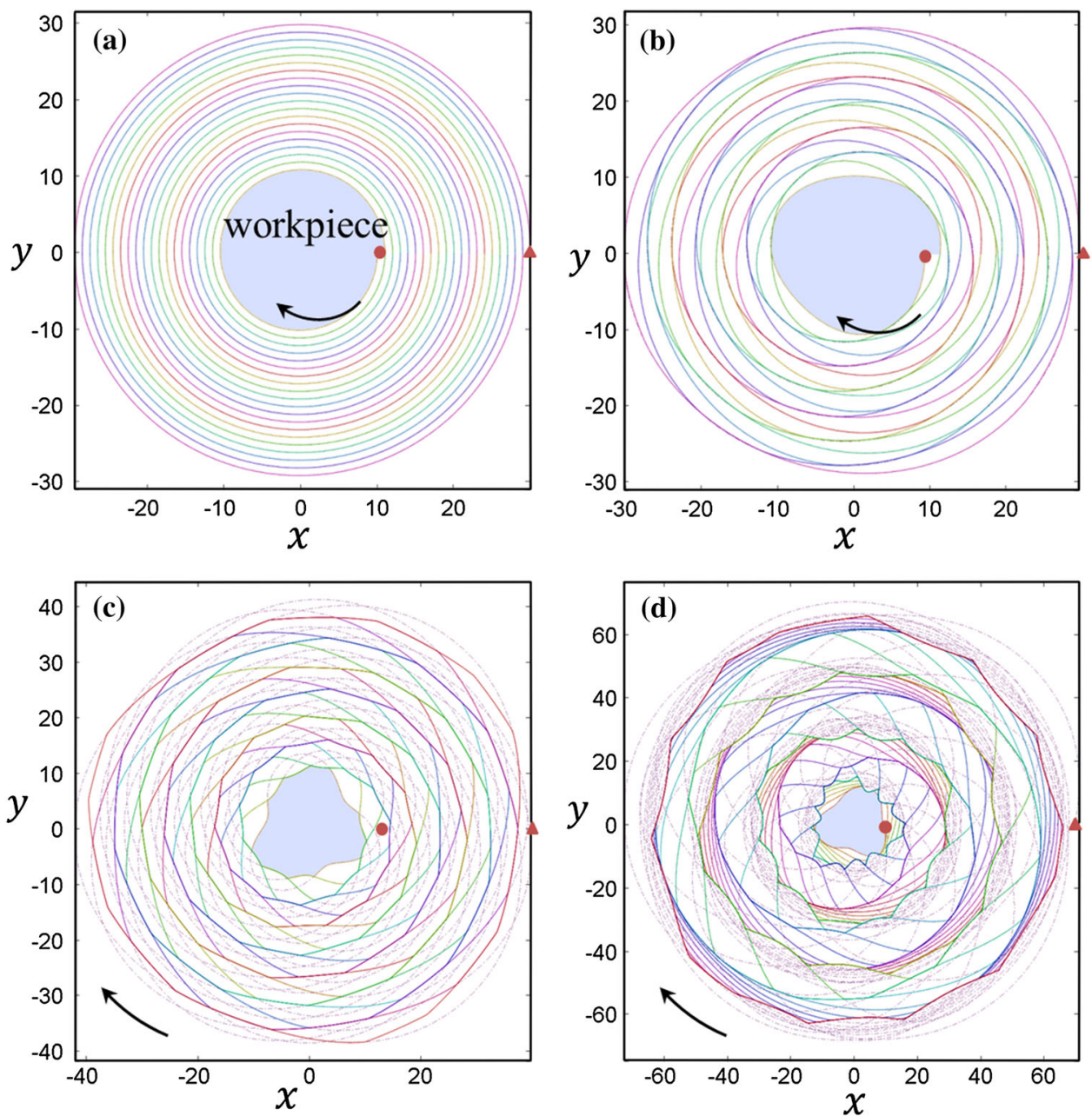

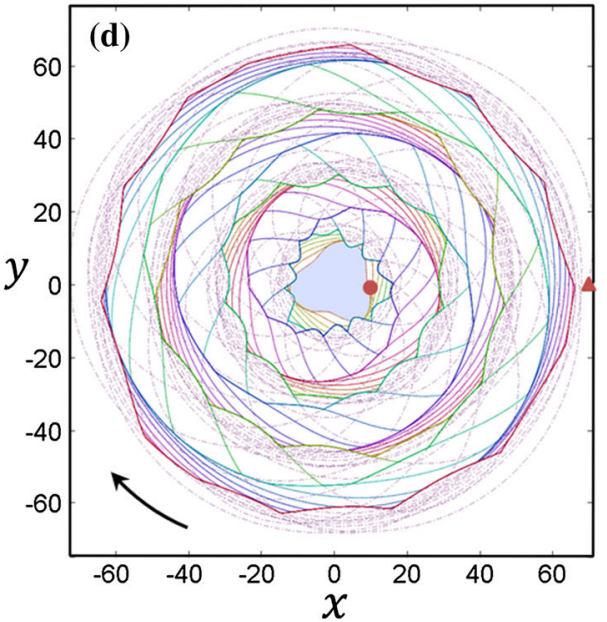

workpiece surface is illustrated in Fig. 7. Solid lines indicate the relative position of the cutting edge while in contact with the work surface and dashed lines indicate that the tool has lost contact with the surface in Fig. 7. As $\kappa$ increases, the system undergoes a series of motions ranging from stable cutting to periodic motion to quasi-periodic motion, and eventually, culminating in chaotic motions. For $\kappa=0.1$, the system operating point is located in the stable region of Fig. 3, and the cutter vibration converges to zero (Figs. 5a, 6a) and the cutting edge trajectory on the workpiece surface is an isometric helix which indicates a smooth workpiece surface, as shown in Fig. 7a. For $\kappa=0.12$, the system parameters are located in an unstable region but very close to the stability boundary. Initial conditions at this point diverge and lead to a periodic motion after a long transient time, as shown in Fig. 5b. In the corresponding Fourier transform shown in Fig. 6b, there is a base frequency component at about $f_{0} \approx 0.17$ and a small-amplitude peak occurs at about 0.34 . This spectrum indicates that the system exhibits periodic motion. Since $\kappa$ here is a critical value that is close to the stability boundary, it is expected that the nonlinearities due to the loss of contact do not significantly affect the sys- tem response, and the trajectory of the cutter on the surface almost coincides with the updating of workpiece as shown in Fig. 7b. When $\kappa=0.42$, the amplitude of vibrations is increased and the tool experiences a quasi-periodic motion in which there is low frequency modulation, as shown in Fig. $5 \mathrm{c}$. The frequency modulation can be seen more clearly in Fig. $6 c$. The carrier wave $f_{0} \approx 0.177$ is modulated by lowfrequency oscillations at $f_{s} \approx 0.0061$. The small-amplitude peaks $f_{0} \pm f_{s}$ next to the base frequency $f_{0}$ result from the frequency modulation. Since the ratio of $f_{0} / f_{s}$ is an irrational number, the system experiences a quasi-periodic motion. At the same time, the dashed lines are no longer coincident with the solid lines in Fig. 7c, due to the strong effects from loss of contact. To generate the results of Fig. $5 \mathrm{~d}, \kappa$ is increased further to 0.7 and the system goes from a quasi-periodic state to a chaotic state. The Fourier transform at this parameter value is no longer discrete but exhibits a continuous spectral character, as shown in Fig. 6d. This verifies the aperiodic motion of the system. Furthermore, the cutting chips are not distributed evenly during this motion, as shown in Fig. 7d.

To further illustrate the multiple regenerative effect on the surface finish of the workpiece, an expanded section of 


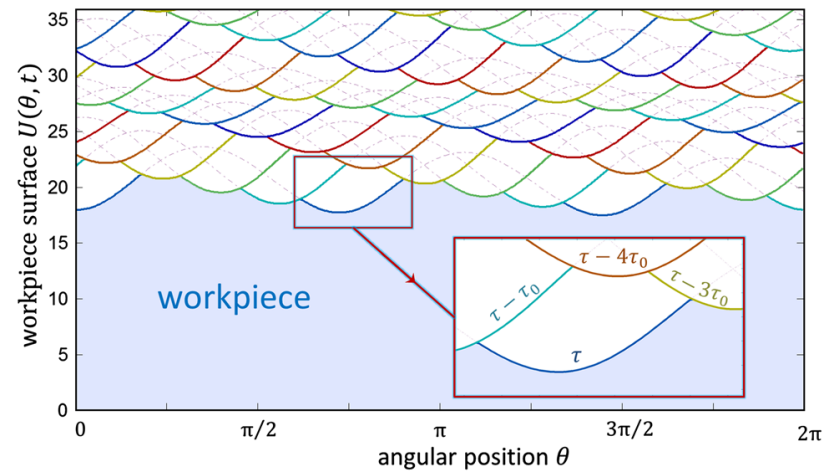

Fig. 8 Expanded workpiece surface of Fig. 7c along $\theta$ direction, with the expanded figure in the inset illustrating mutiple regenerative cutting

Fig. 7c along the $\theta$ direction is provided in Fig. 8. As illustrated in the subplot of Fig. 8, each chip is formed by the position of cutter at the current time $\tau$, one preceding period $\tau-\tau_{0}, \tau-4 \tau_{0}$ and $\tau-3 \tau_{0}$ preceding periods. In this case, the time delay is no longer a single constant but a variable which is the so called variable-integer delay or variabledigital delay in digital circuits and data acquisition processes (e.g., $[29,30])$. The variable-integer delays in Fig. 9 correspond to the the simulation results shown in Figs. 5 and 7, with the time delay being defined as zero during the loss of contact duration of the cutter. In the case of stable regenerative cutting, the time delay is a constant $\tau_{0}$ which is equal to the period of rotation as shown in Fig. 9a. For the periodic motions shown in Figs. $5 \mathrm{~b}$ and $7 \mathrm{~b}$, loss of contact is present and the variable-integer delay is an integer quantity
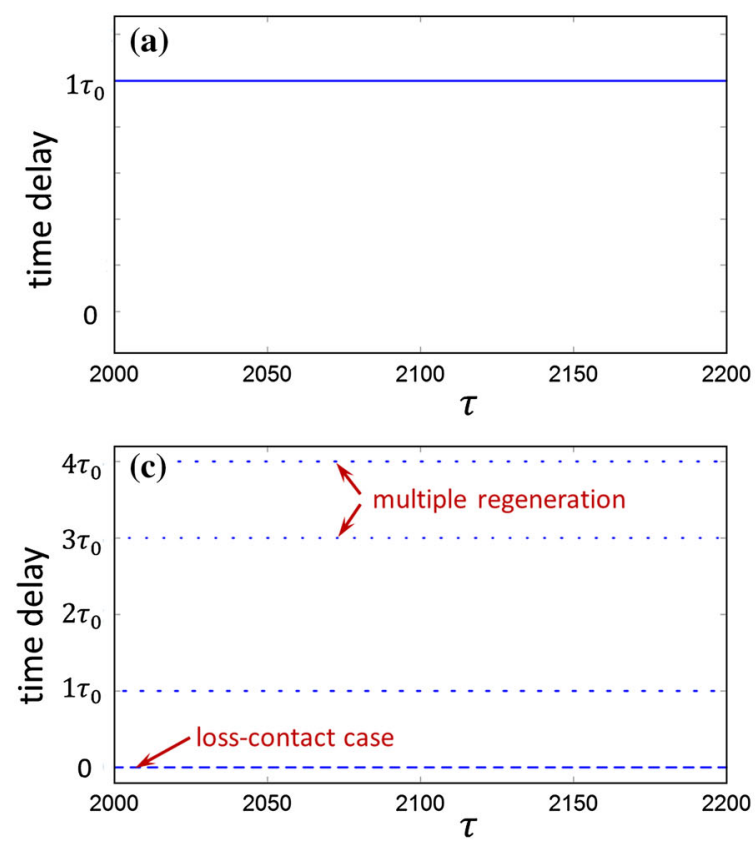

that is equal to either $\tau_{0}$ or $2 \tau_{0}$ as shown in Fig. 9b. Here, the duty cycle of loss of contact has a short duration since $\kappa=0.12$ is a critical value for unstable cutting. Increasing $\kappa$ further, as shown in Fig. 9c, d, the variable-integer time delay becomes more complex and is spread over a wide range from $\tau_{0}$ to $12 \tau_{0}$. At the same time, the duration of loss of contact becomes long. Due to this large variation in the time delay, the approach used to study multiple regenerative cutting in previous work $[3,6]$ will not be effective here due to strong loss of contact effects.

\subsection{Bifurcations and route to chaos}

As the stability of the multiple regenerative cutting system is dependent on the driving speed $\omega$ and the coefficient $\kappa$ which is related to the axial cutting depth, additional parametric studies are carried out to understand this dependence. Since the map has $m+2$ dimensions, a three-dimensional phase space is reconstructed by using an additional pseudostate $x\left(\tau-\tau_{0}\right)$ [27], as illustrated in Fig. 10. The attractor realized in Fig. $5 \mathrm{c}$ is a two-dimensional torus, which follows as a consequence of a subcritical Neimark-Sacker bifurcation. To further explore the system's nonlinear behavior, a $m+1$ dimensional Poincaré section, which is defined by $\dot{x}(\tau)=0$ is considered and illustrated in Fig. 10a. The map may have additional solutions due to the high dimension of the system. For instance, when $\omega=2$ and $\kappa=22$, the system response can be a quasi-periodic motion and the attractor is a limit 2-torus which means there are two incommensurate
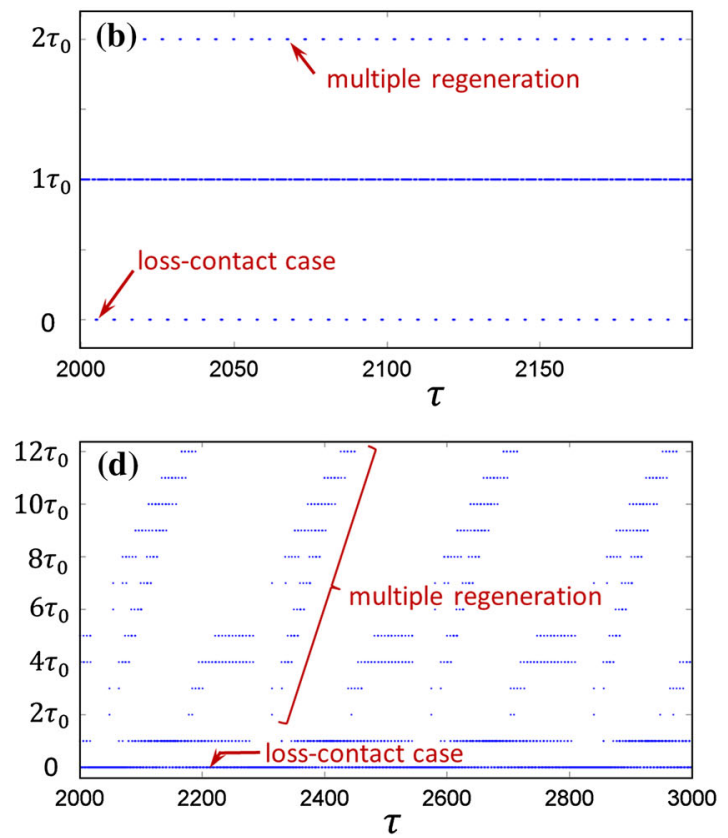

Fig. 9 Variable-integer time delays corresponding to the simulation results of Figs. 5 and 7 at $\omega=0.4$ : a $\kappa=0.1, \mathbf{b} \kappa=0.12$, c $\kappa=0.42$, and d $\kappa=0.7$ 
Fig. 10 Attractors and Poincaré section in the reconstructed phase space: a attractor obtained through the simulations corresponding to Fig. $5 \mathrm{c}$ and Poincaré section defined by $\dot{x}(\tau)=0$ and $\mathbf{b}$ multiple solutions corresponding to different initial condition at $\omega=2$ and $\kappa=22$
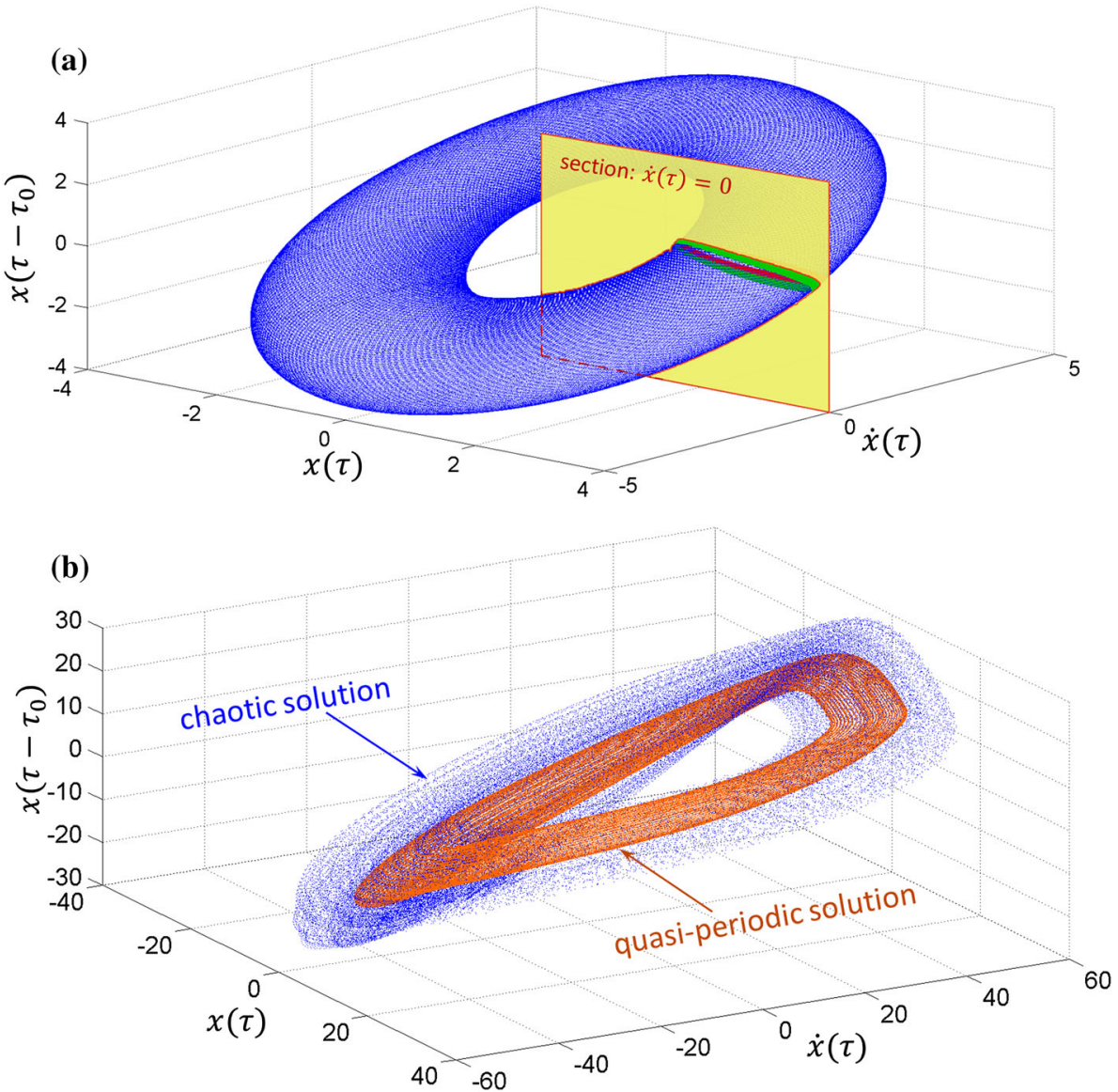

frequencies associated with the response. The corresponding motion is illustrated in Fig. 10b. For different initial conditions and the same parameter values of $\omega$ and $\kappa$, the system can also exhibit chaotic motion, as depicted in Fig. 10b. Since there are $m+2$ states used to describe the map, a small random perturbation $\left(\left\|y_{1}\right\| \ll 1\right)$ is used to define the initial condition. Noting that the trivial solution $y_{i} \equiv 0$ is also a possibility, the system has at least three solutions for this set of parameter values.

For different driving speeds $\omega$, bifurcation diagrams constructed by using the cutting coefficient $\kappa$ as a control parameter are shown in Fig. 11. Both forward and backward sweeps of $\kappa$ are used here in order to examine multiple solutions. The bifurcation diagrams indicate that as the coefficient $\kappa$ is quasi-statically increased, the system goes from periodic motion to chaotic and hyperchaotic motion through subcritical Neimark-Sacker bifurcations. On one of the routes, a Hopf bifurcation occurs followed by a Neimark-Sacker bifurcation leading to quasi-periodic motion, and subsequently, to chaos and hyperchaos from there. From Fig. 11b, it can also be discerned that the system has different solutions for forward and backward sweeps when $\omega=2$ and $\kappa \epsilon$ $(14,23)$. At a drive speed of $\omega=5$, which corresponds to a low driving speed in an oil and gas drilling operation, a period doubling route to chaos is realized as shown in Fig. 11c.
To illustrate the complex response structure influenced by the multiple regenerative cutting process, some attractors and their Poincare intersections are plotted for different parameters and rendered in light and shadow in Figs. 12, 13, 14 and 15. The chaotic attractor shown in Fig. 12a corresponds to the simulation result of Fig. 5d, and this attractor comes into being following a Neimark-Sacker bifurcation. A map of the Poincaré intersections constructed from Fig. 12a is given in Fig. 12b, and these intersections reveal the fractal structure of the attractor. As the $\kappa$ is increased to 1.3 in Fig. 12c, the attractor becomes more complex and the Poincare intersections appear to be spread out in a random manner. This is infact associated with a hyperchaotic behavior of a highdimensional attractor, whose presence is confirmed by computing the Lyapunov exponents in the next subsection.

Two different attractors obtained at $\omega=2$ and $\kappa=22$, with different initial conditions are shown in Fig. 13. For the quasi-periodic case (Fig. 13a,b), the map of Poincaré intersections of the torus is a continuous array of intersections on a closed curve. On the other hand, when the system undergoes a chaotic motion (Fig. 13c,d), the map of Poincaré intersections reveals a fractal structure. For a drive speed of $\omega=5$, setting $\kappa=17$, the system response is found to become chaotic and the associated map of Poincaré intersections has a fractal dimension larger than 2; the associated 
(a)
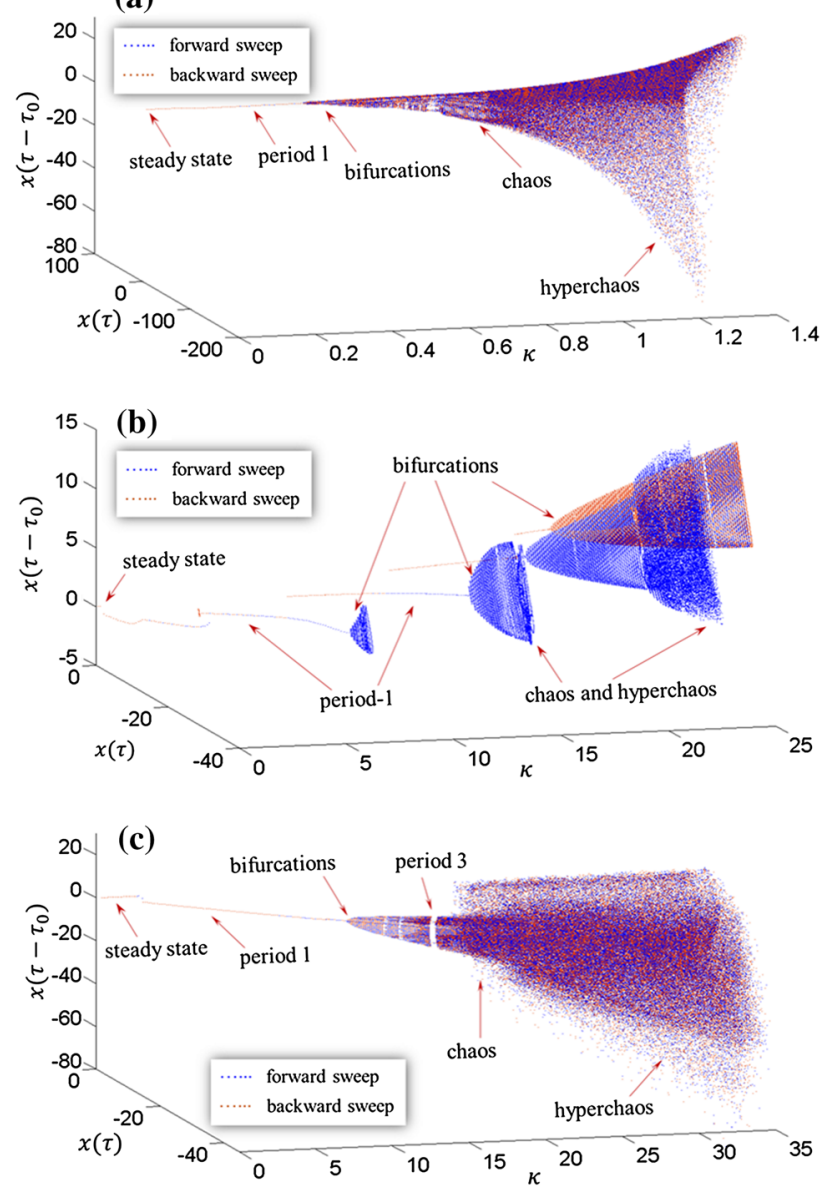

Fig. 11 Bifurcation diagram on Poincaré section $\dot{x}(\tau)=0$ for different driving speeds: $\mathbf{a} \omega=0.4, \mathbf{b} \omega=2$, and $\mathbf{c} \omega=5$. The blue points correspond to forward quasi-static sweep of $\kappa$ while the orange points correspond to backward quasi-static sweep of $\kappa$. (Color figure online)

plots are depicted in Fig. 14a,b. As $\kappa$ is increased to 20, as shown in Fig. 14c,d, the system exhibits a chaotic response, with the corresponding strange attractor having a high dimension. The map of Poincaré intersections which nearly fills the region is found to have a fractal dimension higher than three. When $\kappa$ is increased to 28 as shown in Fig. 14e,f, the system experiences a hyperchaotic motion, a behavior associated with more than one positive Lyapunov exponent. In this case, the attractor has a fuzzy appearance and its corresponding map of Poincaré intersections resembles a collection of densely packed random points. Furthermore, the amplitude of vibrations in the hyperchaotic state (Fig. 14e) is much higher than that observed in the chaotic state (Fig. 14a,c). For a drive speed of $\omega=12$, which is an appropriate speed for drilling process, the chaotic attractor is formed via both period-doubling and Neimark-Sacker bifurcations. It is noted that this route to chaos is different from those associated with the results shown in Fig. 15. Additionally, it is interesting to note the three half-twists on this attractor, chracteristics similar to that of a Möbius strip in topology.

\subsection{Chaotic behavior}

To identify chaotic and hyperchaotic behavior of the $m+2$ dimensional system $(m=600)$ subjected to multiple regenerative cutting effects, both the correlation dimension and Lyapunov spectra calculations are carried out. The correlation dimension is defined as $[31,27]$

$D_{c}=\lim _{n \rightarrow \infty} \lim _{r \rightarrow 0} \frac{\log C(r)}{\log r}$

where $n$ is the total number of data points, $r$ is the radius of a hyper sphere and $C(r)$ is the correlation integral function.

By using the time series data $x(\tau)$, the pseudo-state space is reconstructed based on the so-called delayed coordinates [27]. The delayed coordinates serve as a better measure of reconstructing the state-space since the $u_{i}(\tau)$ are non-smooth and dependent on the resolution (parameter $m$ ) of the semidiscretization method. The plots used for the correlation dimension calculation of the attractor of Fig. 14a is shown in Fig. 16. As the embedding dimension is $d$ increases, the plots become parallel in the scaling region and the slopes converge to the correlation dimension value of $D_{c}=2.41$. Other dimension calculation results obtained for the attractors shown in Figs. 12, 13, 14 and 15 are listed in Table 1. Here, the authors use about $n=81,000$ data points for the correlation dimension calculation of each attractor shown in Table 1. Since the time series of $x(\tau)$ may be correlated with the the preceding position cutter $x\left(\tau-l \tau_{0}\right)$, where $l$ can be higher than 10 due to the strong multiple regenerative effects, the embedding dimension $d$ has to be larger than $l$. When the attractor is high-dimensional, convergence is not realized even when the embedding dimension is increased to 30. For the correlation dimension results shown in Table 1, $D_{c}$ is estimated as the mean of 10 different calculations carried out by using random initial conditions on the attractor. The standard deviations obtained for each of these computations are also shown in the table. The cases for which the correlation dimensions are shown in parenthesis in Table 1 are cases for which the correlation dimension calculations do not converge well.

For the high-dimensional piecewise linear map system Eq. (13), the Lyapunov spectrum with $m+2$ exponents are calculated by using the method proposed by Wolf et al. [32] The Lyapunov spectrum obtained for the attractors of Figs. 14c and 15a are shown in Fig. 17. Here, the Lyapunov exponents are ordered so that $\lambda_{1} \geq \lambda_{2} \geq \lambda_{3} \ldots \lambda_{m+2}$. Since the dimension of the original PDE-ODE system is infinite, the Lyapunov spectrum with 602 exponents calculated in order closely approximate a continuous curve. This Lyapunov spectrum can be used to obtain the Lyapunov dimension, which is the so-called Kaplan-Yorke dimension $D_{K Y}$ $[33,34]$. This dimension is defined as 
Fig. 12 Chaotic attractor (a) and hyperchaotic attractor (c) and their corresponding maps of Poincaré intersections $(\mathbf{b}, \mathbf{d})$ of the multiple regenerative cutting system at drive speed $\omega=0.4$ : a, $\mathbf{b} \kappa=0.7$, chaotic response and $\mathbf{c}, \mathbf{d} \kappa=1.3$, hyperchaotic response. The dashed straight lines on the attractors indicate the Poincare sections associated with the Poincaré intersections

Fig. 13 Two attractors (a) and (c) and their correspondings maps of Poincaré intersections (b) and (d) realized at drive speed $\omega=2$ and $\kappa=22$ for different initial condition: $\mathbf{a}, \mathbf{b}$ quasi-periodic motion and limit torus attractor and $\mathbf{c}, \mathbf{d}$ chaotic motion and strange attractor
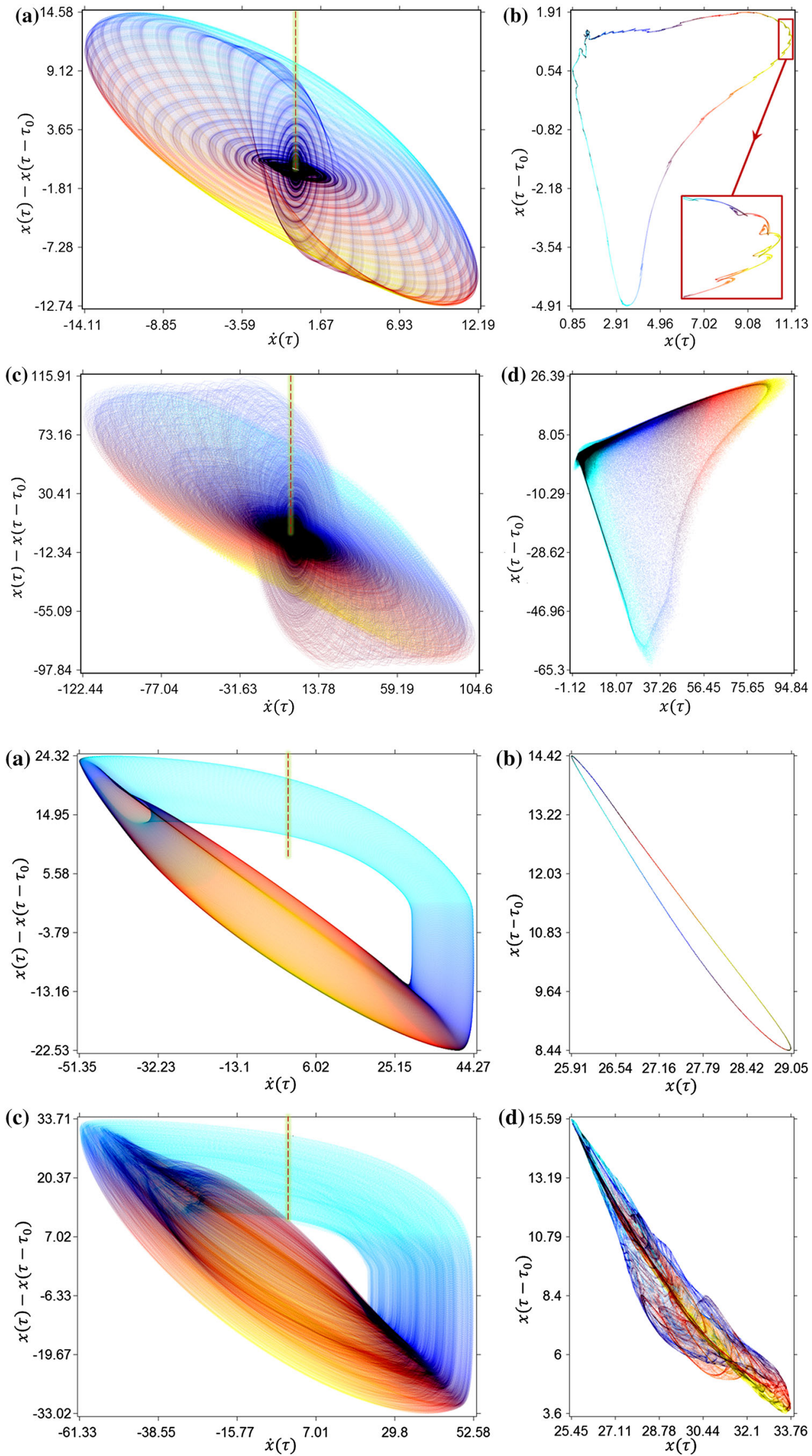
Fig. 14 Chaotic attractor (a) and hyperchaotic attractor (c) and their corresponding maps of Poincaré intersections $(\mathbf{b}, \mathbf{d})$ at drive speed $\omega=5: \mathbf{a}, \mathbf{b} \kappa=17$, chaotic attractor, $\mathbf{c}, \mathbf{d} \kappa=20$, high-dimensional chaotic attractor, and $\mathbf{e}, \mathbf{f} \kappa=28$, hyperchaotic attractor
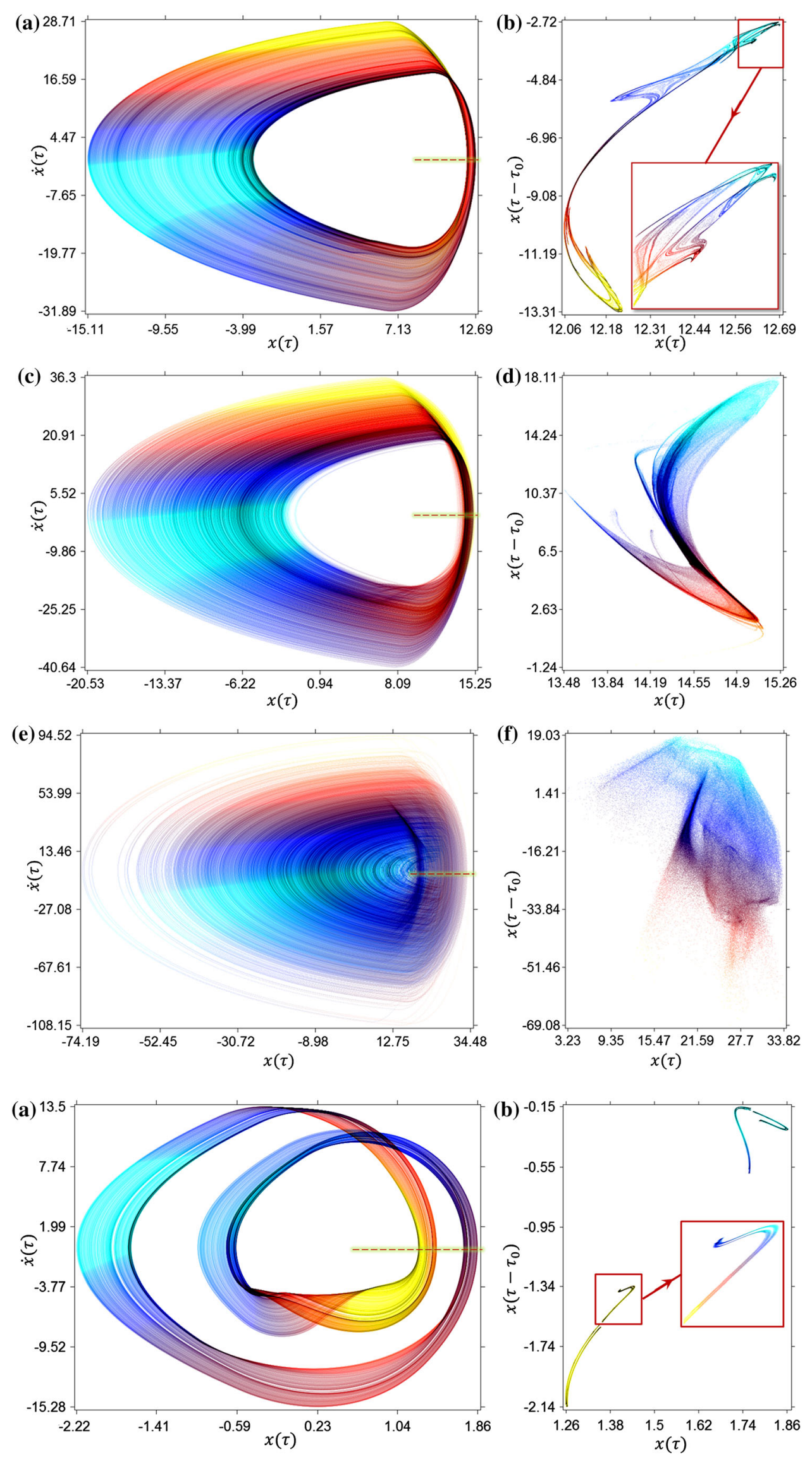

Fig. 15 Chaotic attractor (a) and corresponding map of Poincaré intersections (b) for drive speed $\omega=12$ and $\kappa=54$ 


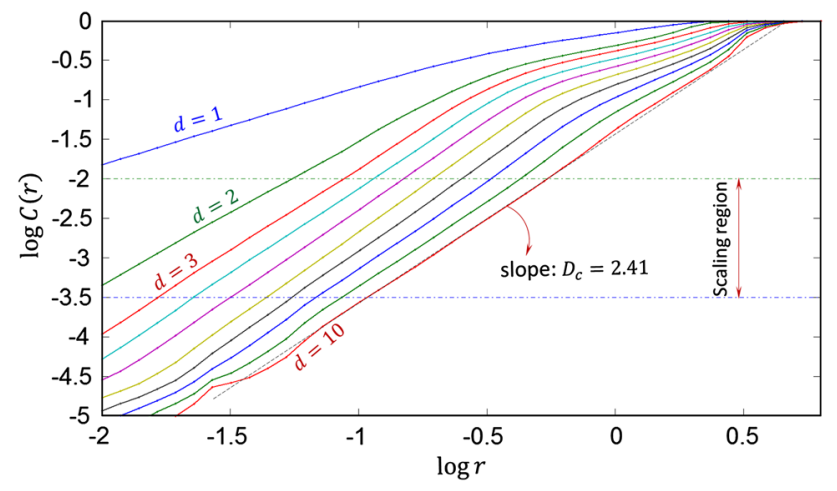

Fig. 16 Plots for computation of correlation dimension $D_{c}$ from time series data of $x(t)$ for the attractor of Fig. 12a. As the embedding dimension $d$ is increased, the slopes in the linear zone converge, and from the scaling region, the correlation dimension is determined as $D_{c}=2.41$

$D_{K Y}=k+\frac{\Lambda_{k}}{\left|\lambda_{k+1}\right|}$

where the scalar $\Lambda_{k}$ is the value of $\Lambda_{i}$ when $i=k$. And $\Lambda_{i}$ is cumulative sum of $\lambda_{i}$ which it is defined as

$\Lambda_{i}=\sum_{j=1}^{i} \lambda_{j}$

and $k$ in Eq. (24) is a constant integer number that when $i=k, \Lambda_{i} \geqslant 0$, and while $i=k+1, \Lambda_{i}<0$. As shown in Fig. 17, $k=3$ here for this Lyapunov spectrum.

The Lyanpunov exponents used to calculate the KaplanYorke dimension for the chaotic attractor of Fig. 15a are illustrated in the inset of Fig. 17. The system has one positive Lyapunov exponent for the parameter values $\omega=12$ and $\kappa=54$. The zero point of $\Lambda_{k}$, which indicates the KaplanYorke dimension, is obtained and found to be $D_{K Y}=3.18$. Computations of the Kaplan-Yorke dimension are carried out for the attractors shown in Figs. 12, 13, 14 and 15 as shown in the Table 1. Here, for this high-dimensional nonlinear system, the Kaplan-Yorke dimension computations are found to show better convergence than the correlation dimension computations. It is interesting to note that there is no zero
Lyapunov exponent for the hyperchaotic attractor case of Fig. 12c, which is anomalous for time-continuous systems. This can be explained as follows. When the system undergoes hyperchaotic motion at $\omega=0.4$, strong beat vibrations occur and the trajectory of the system goes from the center of the attractor to the boundary in a spiral motion and later returns to the center. When the motion is expanding outwards toward the boundary, any two trajectories with an infinitesimally small separation along the direction of trajectories will diverge. On the other hand, the two trajectories will converge when the motion is contracting. Instead of a zero local Lyapunov exponent, a positive or negative exponent exists along the direction of trajectory in this motion. When the system goes into chaos or hyperchaos, principal directions are effected by the divergence along the direction of trajectory and there are no zero Lyapunove exponents.

Additionally, the Lyapunov spectra and Kaplan-Yorke dimensions for both forward and backward sweep of $\kappa$ are computed. The variation of the first ten Lyapunov exponents as $\kappa$ is varied are shown in Fig. 18a. Here, the authors focus on the interval of $\kappa$ that might lead to complex dynamics according to the Fig. 11. In Fig. 18b, the authors give the Kaplan-Yorke dimensions computed for forward and backward quasi-static sweeps of $\kappa$, at the drive speed $\omega=0.4$. For $\kappa<0.41$, the Lyapunov exponents are $(0,-,-, \ldots)$, which indicates the system exhibits a periodic motion and the attractor is a limit cycle. For $\kappa \in(0.41,0.68)$, the Lyapunov exponents change to $(0,0,-, \ldots)$, which may lead to a quasi-periodic motion; the attractor is a torus with a dimension of two. It is worth mentioning that a limit cycle also coexists in this interval of $\kappa$. As $\kappa$ is increased, the system goes to chaos or hyperchaos and the Kaplan-Yorke dimensions of these strange attractors increase to approximately 8 . For $\kappa>1$, there are three positive Lyapunov exponents but no zero exponent because of the strong hyperchaotic motion with beat characteristics. Additionally, there is only one solution at this driving speed, which is consistent with the bifurcation diagram of Fig. 11a. The same computations are carried out for the drive speed of $\omega=2$, as shown in Fig. 19. As $\kappa$ is increased, the system undergoes periodic, quasi-periodic, chaotic, and hyperchaotic motions, and the dimension of the
Table 1 Correlation dimension $D_{c}$, Kaplan-Yorke dimension $D_{K Y}$ and Lyapunov exponents for the attractors shown in Figs. 12, 13, 14 and 15

\begin{tabular}{lllll}
\hline Attractor & $(\omega, \kappa)$ & $D_{c}$ & $D_{K Y}$ & Lyapunov exponents \\
\hline Fig. 12a & $(0.4,0.7)$ & $2.41 \pm 0.05$ & $2.47 \pm 0.01$ & $(+, 0,-,-, \ldots)$ \\
Fig. 12c & $(0.4,1.3)$ & $(5.6)$ & $7.25 \pm 0.08$ & $(+,+,+,-, \ldots)$ \\
Fig. 13a & $(2.0,22)$ & $2 \pm 0.03$ & $2 \pm 0.001$ & $(0,0,-,-, \ldots)$ \\
Fig. 13c & $(2.0,22)$ & $2.14 \pm 0.05$ & $2.17 \pm 0.02$ & $(+, 0,-,-, \ldots)$ \\
Fig. 14a & $(5.0,17)$ & $2.59 \pm 0.1$ & $2.64 \pm 0.01$ & $(+, 0,-,-, \ldots)$ \\
Fig. 14c & $(5.0,20)$ & $(3.61)$ & $3.88 \pm 0.01$ & $(+, 0,-,-, \ldots)$ \\
Fig. 14e & $(5.0,28)$ & $(4.17)$ & $4.90 \pm 0.05$ & $(+,+, 0,-, \ldots)$ \\
Fig. 15a & $(12,54)$ & $2.71 \pm 0.3$ & $3.18 \pm 0.01$ & $(+, 0,-,-, \ldots)$ \\
\hline
\end{tabular}


Fig. 17 Lyapunov spectrum of system with 602 dimensions (Eq. (13)), with the inset containing the details of the procedure used to compute the Kaplan-Yorke dimension

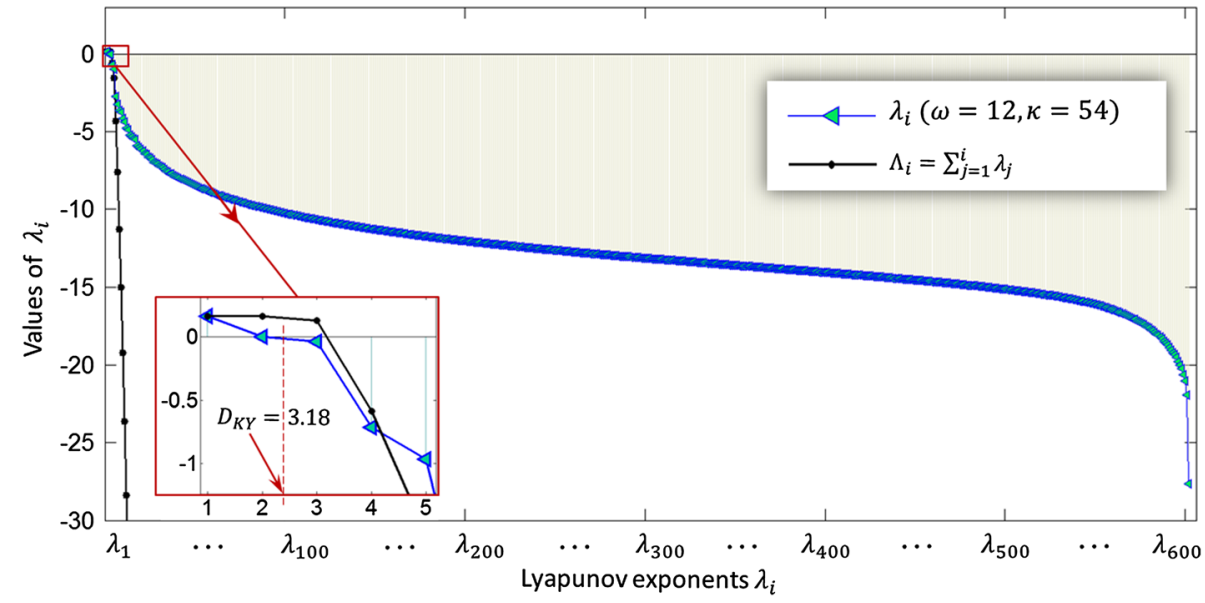

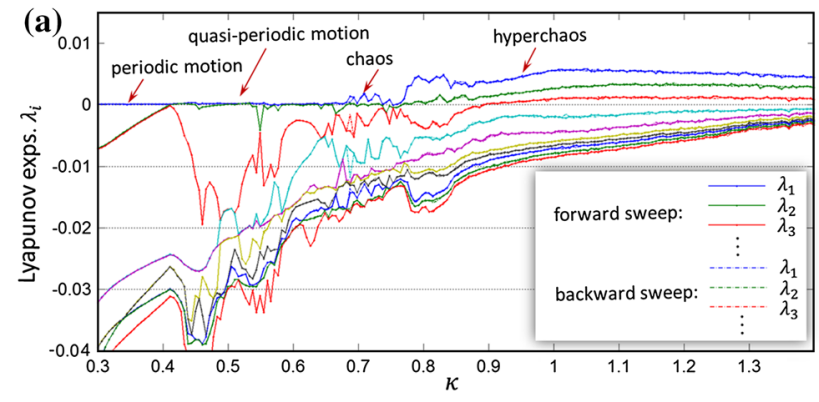

(b)

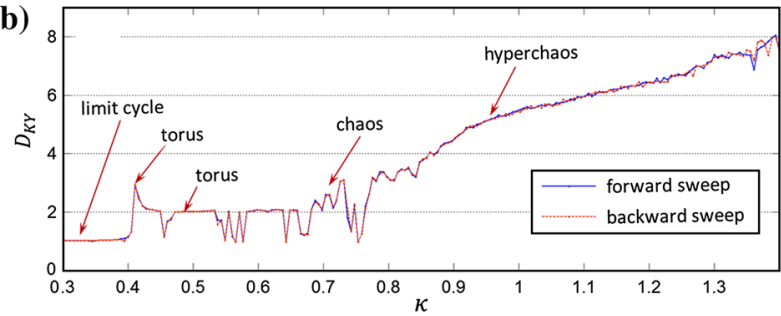

Fig. 18 Parametrical study at driving speed $\omega=0.4$ : a the ten lagest Lyapunov exponents $\lambda_{1}-\lambda_{10}$ versus $\kappa$ and $\mathbf{b}$ Kaplan-Yorke dimension $D_{K Y}$ versus $\kappa$

attractor goes from 1 to approximately 4.5 (Fig. 11b). Here, the results obtained for the forward sweep and backward sweep do not coincide with each other. For example, in the interval $\kappa \in(14,21)$, the system exhibits periodic motion and quasi-periodic motion or chaotic motion for the same parameter values but different initial conditions. In the interval $\kappa \in(21,23.2)$, starting at different initial conditions, the system response state can be a quasi-periodic motion or a chaotic motion or a hyperchaotic motion. These results are also consistent with the bifurcation diagram Fig. 11b.

\section{Concluding remarks}

In this article, the authors have studied a fundamental nonlinear cutting process with loss of contact effects. To focus
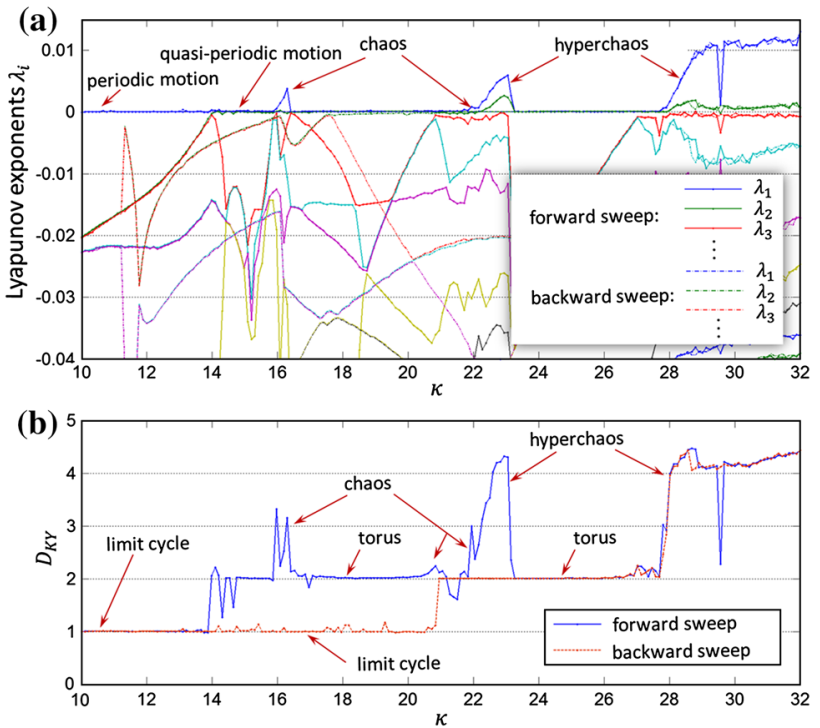

Fig. 19 Parametrical study at driving speed $\omega=2$ : a the ten lagest Lyapunov exponents $\lambda_{1}-\lambda_{10}$ versus $\kappa$ and $\mathbf{b}$ Kaplan-Yorke dimension $D_{K Y}$ versus $\kappa$

on multiple regenerative effects, a simplified one degreeof-freedom tool model governed by an ODE is used. To capture the multiple regenerative effects, instead of using DDE, a PDE is used to describe the updating of the workpiece based on previous studies. Thus, this PDE-ODE model represents an exact geometrical updating of workpiece and the time delay can be any integer multiple of the toothpass period (i.e., $\tau_{0}, 2 \tau_{0}, 3 \tau_{0} \ldots$ ) which is the so called variable-integer delay.

Analysis of this PDE-ODE model indicates that the loss of contact effects introduces two influences on the system dynamics. One of them is the non-smooth (piecewise linear) cutting force which is captured in the ODE. The other is the multiple regenerative effects, which is captured through switching boundary conditions of the PDE. To solve this nonlinear PDE-ODE system, a reduction based on semi- 
discretization method is used and a discrete-time mapping system is obtained. In the development of this reduction, when the workpiece surface description (PDE) is discretized into $m$ elements, the mapping system is of $m+2$ dimensions. This map is high dimensional and continuous but piecewise linear, and it can be regarded as a high-dimensional tent map. For numerical studies of this map, parameter values have been selected to reflect both metal cutting and oil drilling operations. The simulations show that both the non-smooth cutting force and multiple regenerative effect can restrain the divergent behavior of the corresponding linear system and lead to nonlinear oscillations. As the dimensionless cutting coefficient $\kappa$ is increased, the system may experience stable cutting, periodic motion, quasi-periodic motion, chaotic motion, and hyperchaotic motion. At the same time, the surface updating of the workpiece becomes more and more complex. Due to the multiple regenerative effect, it is found that the time delay in the system can vary in a wide range from 0 to more than $10 \tau_{0}$.

Parametric studies conducted with the dimensionless drive speed $\omega$ and dimensionless cutting coefficient $\kappa$ as control parameters reveal the rich nonlinear dynamics of the system, including bifurcations and routes to chaos that can be experienced by the system. By carrying forward and reverse quasistatic sweeps of $\kappa$, it is found that multiple responses exist for certain parameter values. The rendered attractors and their corresponding maps of Poincaré intersections help understand the attractor structures and identify torus attractors, chaotic attractors, and hyperchaotic attractors. Both correlation dimension and Kaplan-Yorke dimension are computed, and from these computations, it is learned that the dimensions of the attractors can be more than 8 as the system moves to hyperchaos. Results obtained for the Lyapunov exponents and Kaplan-Yorke dimension show intervals of limit cycle motions, torus motions, chaotic attractors, and hyperchaotic attractors in the considered range of the cutting coefficient $\kappa$.

The paradigm and analyses presented in this work can be extended to different cutting operations in which multiple regenerative effects play an important role. Furthermore, the reduction outlined in this work for the combined PDE-ODE system can be serve as a template for other such systems.

Acknowledgments The authors from Shanghai Jiao Tong University gratefully acknowledge the support received through 973 Grant No. 2011 CB706803 and NSFC Grant No. 10732060.

\section{References}

1. Tlusty J, Polacek M (1963) The stability of machine tools against self excited vibrations in machining. In Proceedings of the ASME International Research in Production Engineering, Vol 465. Pittsburgh, USA, p 474

2. Tobias SA (1965) Machine-tool vibration. Wiley, New York
3. Tlusty J, Ismail F (1981) Basic non-linearity in machining chatter. CIRP Ann Manuf Technol 30(1):299-304

4. Altintas Y, Budak E (1995) Analytical prediction of stability lobes in milling. CIRP Ann Manuf Technol 44(1):357-362

5. Altintas Y (2000) Manufacturing automation: metal cutting mechanics, machine tool vibrations, and $\mathrm{CNC}$ design. Cambridge Univ Press, Cambridge

6. Balachandran B, Zhao MX (2000) A mechanics based model for study of dynamics of milling operations. Meccanica 35(2):89-109

7. Stépán G (2001) Modelling nonlinear regenerative effects in metal cutting. Philos Trans R Soc Lond A 359(1781):739-757

8. Balachandran B (2001) Nonlinear dynamics of milling processes. Philos Trans R Soc Lond A 359(1781):793-819

9. Pratt JR, Nayfeh AH (2001) Chatter control and stability analysis of a cantilever boring bar under regenerative cutting conditions. Philos Trans R Soc Lond A 359(1781):759-792

10. Zhao MX, Balachandran B (2001) Dynamics and stability of milling process. Int J Solids Struct 38(10):2233-2248

11. Richard T, Germay C, Detournay E (2004) Self-excited stick-slip oscillations of drill bits. Comptes Rendus Mcanique 332(8):619626

12. Wang XS, Hu J, Gao JB (2006) Nonlinear dynamics of regenerative cutting processes: comparison of two models. Chaos Solitons Fractals 29(5):1219-1228

13. Insperger T, Stépán G, Turi J (2007) State-dependent delay in regenerative turning processes. Nonlinear Dyn 47:275-283

14. Long XH, Balachandran B, Mann B (2007) Dynamics of milling processes with variable time delays. Nonlinear Dyn 47:49-63

15. Litak G, Syta A, Wiercigroch M (2009) Identification of chaos in a cutting process by the $0-1$ test. Chaos Solitons Fractals 40(5):20952101

16. Litak G, Schubert S, Radons G (2012) Nonlinear dynamics of a regenerative cutting process. Nonlinear Dyn 69(3):1255-1262

17. Germay C, van de Wouw N, Nijmeijer H, Sepulchre R (2009) Nonlinear drillstring dynamics analysis. SIAM J Appl Dyn Syst $8(2): 527-553$

18. Besselink B, van de Wouw N, Nijmeijer H (2011) A semi-analytical study of stick-slip oscillations in drilling systems. ASME J Comput Nonlinear Dyn 6(2):021006

19. Liu X, Vlajic N, Long XH, Meng G, Balachandran B (2013) Nonlinear motions of a flexible rotor with a dill bit: stick-slip and delay effects. Nonlinear Dyn 72(1-2):61-77

20. Grabec I (1988) Chaotic dynamics of the cutting process. Int $\mathbf{J}$ Mach Tools Manuf 28(1):19-32

21. Bailey JA (1975) Friction in metal machining mechanical aspects. Wear 31(2):243-275

22. Banihasan M, Bakhtiari-Nejad F (2011) Chaotic vibrations in highspeed milling. Nonlinear Dyn 66(4):557-574

23. Wahi P, Chatterjee A (2008) Self-interrupted regenerative metal cutting in turning. Int J Non-Linear Mech 43(2):111-123

24. Dombovari Z, Barton DAW, Wilson RE, Stepan G (2011) On the global dynamics of chatter in the orthogonal cutting model. Int $\mathbf{J}$ Non-Linear Mech 46(1):330-338

25. Insperger T, Stépán G (2002) Semi-discretization method for delayed systems. Int J Numer Methods Eng 55(5):503-518

26. Insperger T, Stépán G (2004) Updated semi-discretization method for periodic delay-differential equations with discrete delay. Int J Numer Methods Eng 61(1):117-141

27. Nayfeh AH, Balachandran B (1995) Applied nonlinear dynamics. Wiley, New York

28. Richard T, Germay C, Detournay E (2007) A simplified model to explore the root cause of stick-slip vibrations in drilling systems with drag bits. J Sound Vib 305(3):432-456

29. Johnson MG, Hudson EL (1988) A variable delay line PLL for CPU-coprocessor synchronization. IEEE J Solid-State Circuits 23(5):1218-1223 
30. Farrow CW (1988) A continuously variable digital delay element. In IEEE International Symposium on Circuits and Systems, p 2641-2645

31. Grassberger P, Procaccia I (1983) Measuring the strangeness of strange attractors. Physica D 9(1):189-208

32. Wolf A, Swift JB, Swinney HL, Vastano JA (1985) Determining lyapunov exponents from a time series. Physica D 16(3):285-317
33. Kaplan J, Yorke JA (1979) Functional differential equations and approximation of fixed points. Lecture notes in mathematics, vol 730. Springer, New York, p 228

34. Farmer JD, Ott E, Yorke JA (1983) The dimension of chaotic attractors. Physica D 7(1-3):153-180 